\title{
Search for a Scalar Component of Weak Interactions
}

\author{
Dalibor Zakoucky \\ Nuclear Physics Institute of ASCR \\ 25068 Rez near Prague, Czech Republic \\ E-mail: zakouckyeujf.cas.cz

\section{for WITCH and ISOLDE collaborations} \\ CERN \\ Geneve, Switzerland
}

Weak interactions are described by the Standard Model which postulates the basic assumption about the pure "V(ector)-A(xial vector)" character of the interaction. Nevertheless, even after half a century of development of the model and experimental testing of its fundamental ingredients, experimental data still allow the existence of other types of weak interactions - e.g. scalar interactions are ruled out only on the $7 \%$ level.

Experimental project WITCH (Weak Interaction Trap for CHarged particles) was set up at the isotope separator ISOLDE at CERN trying to probe the properties of the weak interaction in order to look for their forbidden (scalar, tensor) components or at least significantly improve their current experimental limits. Experimental setup consisting of a combination of 2 Penning traps and retardation spectrometer allows to catch the radioactive nuclei from ISOLDE separator, traps and cools them and lets them decay in rest and then probes the energy spectrum of recoiling nuclei which is sensitive to the character of the weak interaction.

First online measurements with the isotope ${ }^{35} \mathrm{Ar}$ which is very sensitive to a possible admixture of scalar interaction were performed in 2011 and 2012. The current status of the experiment and experimental results as well as results of extensive simulations are presented and discussed here.

Speaker 


\section{Theoretical motivation}

The successful unification of the weak and electromagnetic interactions represented a major breakthrough in our understanding of the weak interaction. Nevertheless, the present Standard Model (SM) is generally believed not to be the ultimate theory of particles and their interactions as it contains too many parameters that have to be determined by experiment, while also some features such as e.g. parity violation and $\mathrm{CP}$-violation are not explained by the model itself. Many experiments, ranging from the low-energy scale of nuclear $\beta$-decay to the very highest energies at colliders, tested the Standard Model searching for possible deviations which could point to possible extensions of the model. The existence of new physics beyond the Standard Model was already proven with the discovery of neutrino oscillations [1]. Apart from increasing the energy that is available in particle collisions at the high energy frontier (LHC collider), the sensitivity to new physics can also be improved by increasing the precision of experiments at lower energies, e.g.. in muon decay and in neutron and nuclear $\beta$-decay.

Several important properties of the weak interaction, such as parity violation [2] and the V-A structure of the interaction [3], were discovered in nuclear $\beta$-decay. Also today new $\beta$-decay experiments continue to be an efficient tool to search for new physics beyond the standard electroweak model. In this approach precision measurements are carried out to determine the parameters related to the structure of the weak lepton-quark current or to search for tiny effects that would be induced by the exchange of new massive particles. Such experiments can explore energy regions and features that cannot be probed at present at high-energy colliders where one searches for the direct production of new massive particles. Both approaches are thus largely complementary in the parameter regions that can be probed.

The basis of the Standard Model as a theory of electroweak interactions is the assumption about the character of the interaction: pure V-A interaction with the maximum parity violation, the other types of interaction (scalar, tensor) are excluded. However, the available experimental data still allow the existence of other types of weak interactions with current upper limits for scalar/ tensor coupling constants in nuclear $\beta$-decay, relative to the vector/axial-vector coupling constants being as high as $7 \%$ [4]. A natural way to extend the Standard Model in order to build a more fundamental theory is to look for the deviations from the Standard Model predictions or to check its basic assumptions as e.g. the pure V-A character of the interaction. This can be done experimentally by measurements of the $\beta-3$ correlation after $\beta$-decay. It turns out that these measurements can be very sensitive to the character of the interaction and with realistic requirements on the precision of the measurement one can look for small admixtures of different types of interactions.

\section{Experiment}

\subsection{Experimental method}

One of the correlations in $\beta$-decay sensitive to the new physics outside of Standard Model which can be experimentally studied is the $\beta-v$ correlation. Since it would be difficult to detect neutrinos in a rather compact low-energy $\beta$-decay experiment the study of emitted neutrinos after the $\beta$-decay is replaced by the study of the recoiling daughter nuclei. However, the low energy of recoiling nuclei $(\sim \mathrm{eV})$ prevents the usage of standard radioactive samples since they 


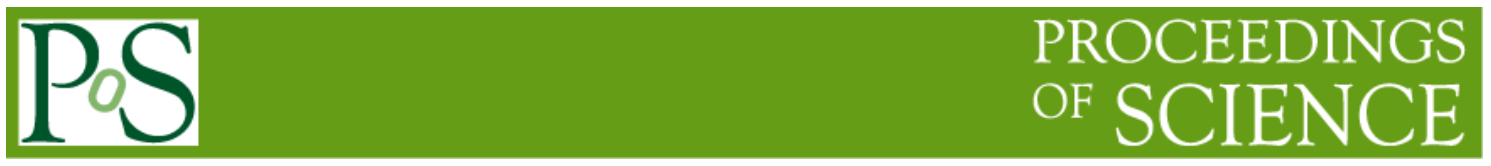

would be absorbed inside the sample material and never escape to be detected. This problem can be solved by using the ion/atom traps where the nuclei can decay in well-localised ion/atom clouds with low energy and low density and recoiling nuclei can escape without significant scattering/absorption.

The shape of the energy spectrum of recoiling nuclei is very different for different decay modes of the weak interaction (see the shapes of the recoil spectra for Scalar and Vector interactions in Fig.1) and therefore by precise experimental determination of the shape of the recoil spectrum one can determine a possible admixture of a (forbidden) scalar component in the (allowed) dominant vector component.

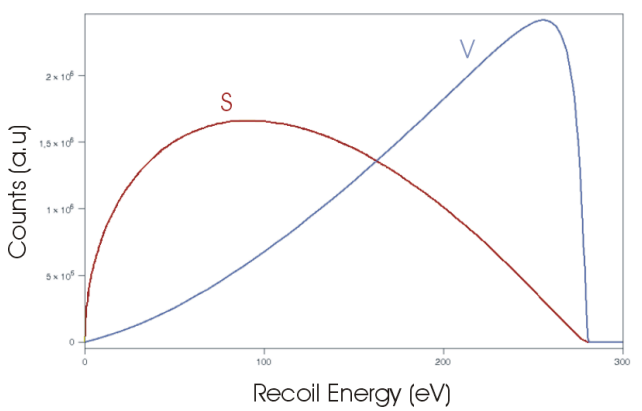

Fig.1 Recoil spectrum for the Vector and Scalar interactions

For precise measurements of the shape of recoil ion energy spectra after $\beta$-decay of trapped radioactive ions the online facility WITCH (Weak Interaction Trap for CHarged particles) has been built at ISOLDE/CERN [5]. This setup uses two Penning traps to catch and trap radioactive ions produced by the ISOLDE separator, cools them and lets them decay as a scattering/absorption free radioactive $\beta$-source and by means of the retardation spectrometer probes the energy spectrum of the recoiling nuclei.

\subsection{Experimental setup}

A scheme of the WITCH setup at ISOLDE [6] is shown in Fig.2. Principal components of the setup are 2 Penning traps and the retardation spectrometer which are mounted inside the big LHe cryostat housing 2 superconducting magnets with fields up to 9T (traps) and 0.2T (spectrometer). Radioactive isotopes to be studied are produced/separated by the ISOLDE separator where the CERN PS booster beam of $10^{13} 1.4 \mathrm{GeV}$ protons/s hits a thick ISOLDE target, the produced isotopes are ionised, extracted and mass separated by ISOLDE separators (General Purpose Separator GPS or High Resolution Separator HRS). Ions from the continuous $30 / 60 \mathrm{keV}$ ISOLDE beam are then stopped, cooled and bunched in a preparatory Penning trap REXTRAP. The cooled and bunched $30 \mathrm{keV}$ beam extracted from REXTRAP is transported through Horizontal Beam Line to WITCH, bent vertically upwards and then slowed down by the Pulsed Drift Tube to $\sim 10^{2} \mathrm{eV}$ [7]. Ions are then trapped in the first Penning trap with pure He buffer gas (cooler trap)

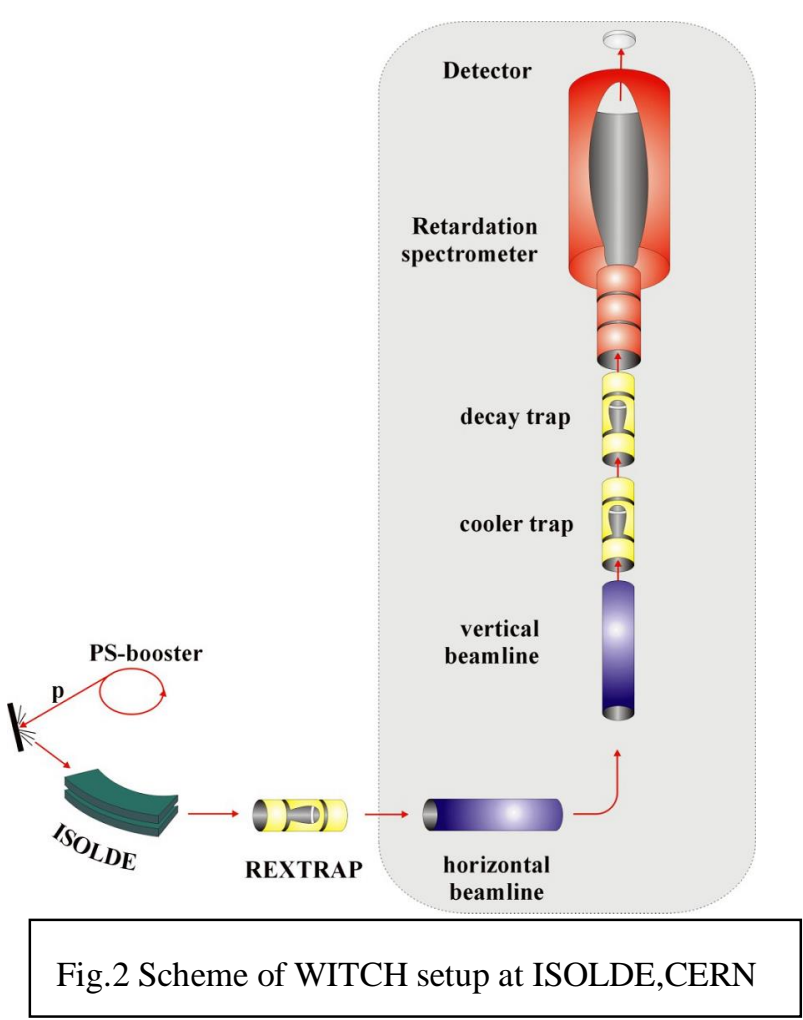


where they are cooled to $\sim 10^{-1} \mathrm{eV}$ and also spatially compressed. Then they are transferred through the narrow pumping diaphragm to the decay trap where they are trapped again and left to decay. The decay trap is pumped to high vacuum in order not to influnce the trapped ions and decay products by collisions with the rest gas. Very narrow diaphragm $(\varnothing=2 \mathrm{~mm})$ and powerfull pumping prohibits leaking of buffer gas from cooler to decay trap. Ions recoiling after the $\beta$ decay in the decay trap are guided by the strong magnetic field (6 or 9T) into the retardation spectrometer (placed in a $0.1 \mathrm{~T}$ magnetic field). Energy spectra of nuclei recoiling after the $\beta$-decay are measured by the combination of a retardation spectrometer (probes the energy) and a MCP detector [8] (counter). The retardation spectrometer blocks the ions with energy below the electrostatic retardation barrier while ions with higher energy get further and are registered by the MCP detector. By variation of the blocking potential we measure the integral recoil spectrum. After passing through the retardation spectrometer the recoiling ions are reaccelerated and detected by the $8 \mathrm{~cm}$ diameter position sensitive MCP detector which serves as a Ăcounterñ

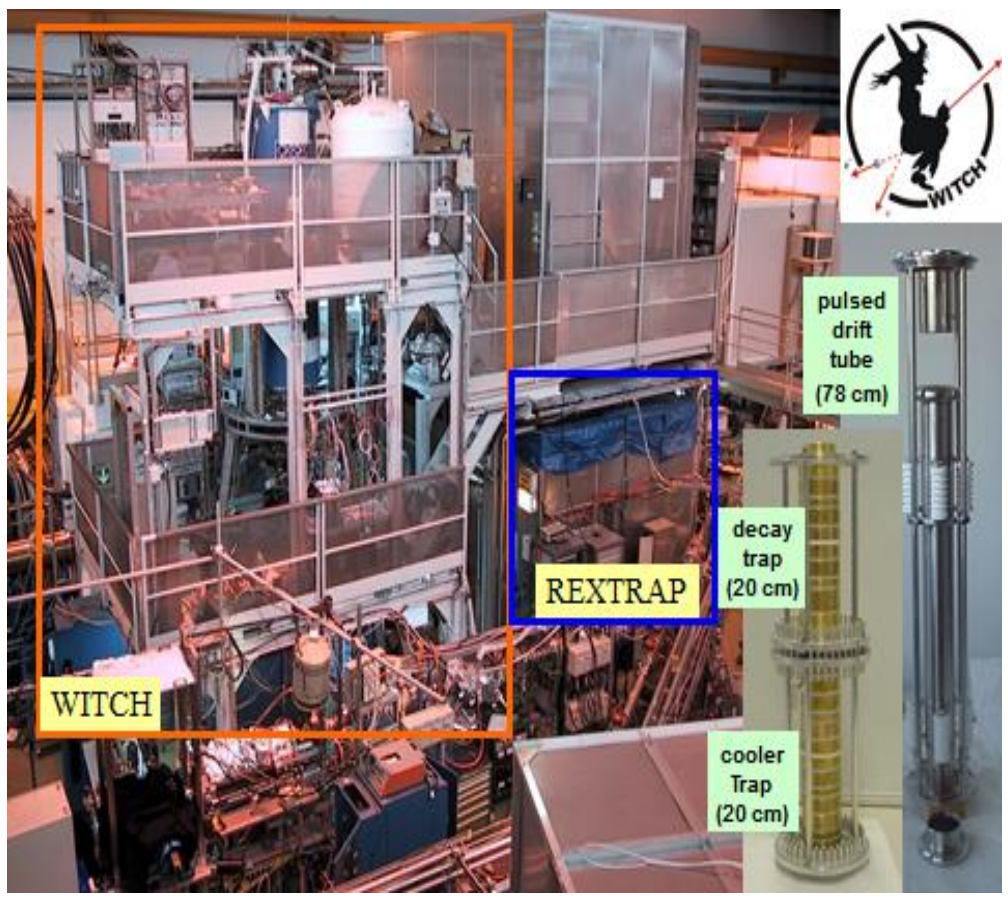
while its position sensitivity enables to record also Ăredundantò information of use for systematic checks.

\section{Experimental data}

The best candidate for a measurement testing the possible existence of a scalar component of weak interactions is the isotope ${ }^{35} \mathrm{Ar}$ which decays by almost pure Fermi decay, while all nuclear structure corrections are small and well calculated and it is also available at ISOLDE and technically feasible. Several online measurements of this isotope were already performed using the WITCH setup.

\subsection{Experiment in 2011}

In 2011 a first successful online experiment with ${ }^{35} \mathrm{Ar}$ was performed where we were able to catch and cool ${ }^{35} \mathrm{Ar}$ ions in the cooler trap to sufficiently low energy, keep them for a long enough time in the decay trap (at rest) and let them decay to form a scattering-free cool 
radioactive $\beta$-source.We unambiguously observed recoil ions after $\beta$-decay of ${ }^{35} \mathrm{Ar}$ and measured the energy spectra of recoiling ions. Statistics was still low but the reasons for that were identified and measures to optimize both the ISOLDE beam and WITCH setup were taken in preparation for the next experiment.

Some experimental data from the online experiment are illustrated in Fig.4. A typical sequence of the measurement is that after the cooled bunch of ions is transferred from the cooler to the decay trap and left to decay, a retardation potential pattern is applied during the $5 \mathrm{~s}$ measurement cycle and recoiling ions after the $\beta$ Ï decay are counted by the MCP detector. After each cycle the trap is cleaned and a new cycle is started. This sequence is repeated for some time to gain statistics. Count rate is high when no retardation of the recoil ions leaving the Penning trap is applied, and low when a retardation voltage is applied (dips in spectrum). The time structure of the retardation voltage is shown in the inset. The decay of the count rate reflects the ${ }^{35}$ Ar half-life of $1.78 \mathrm{~s}$.

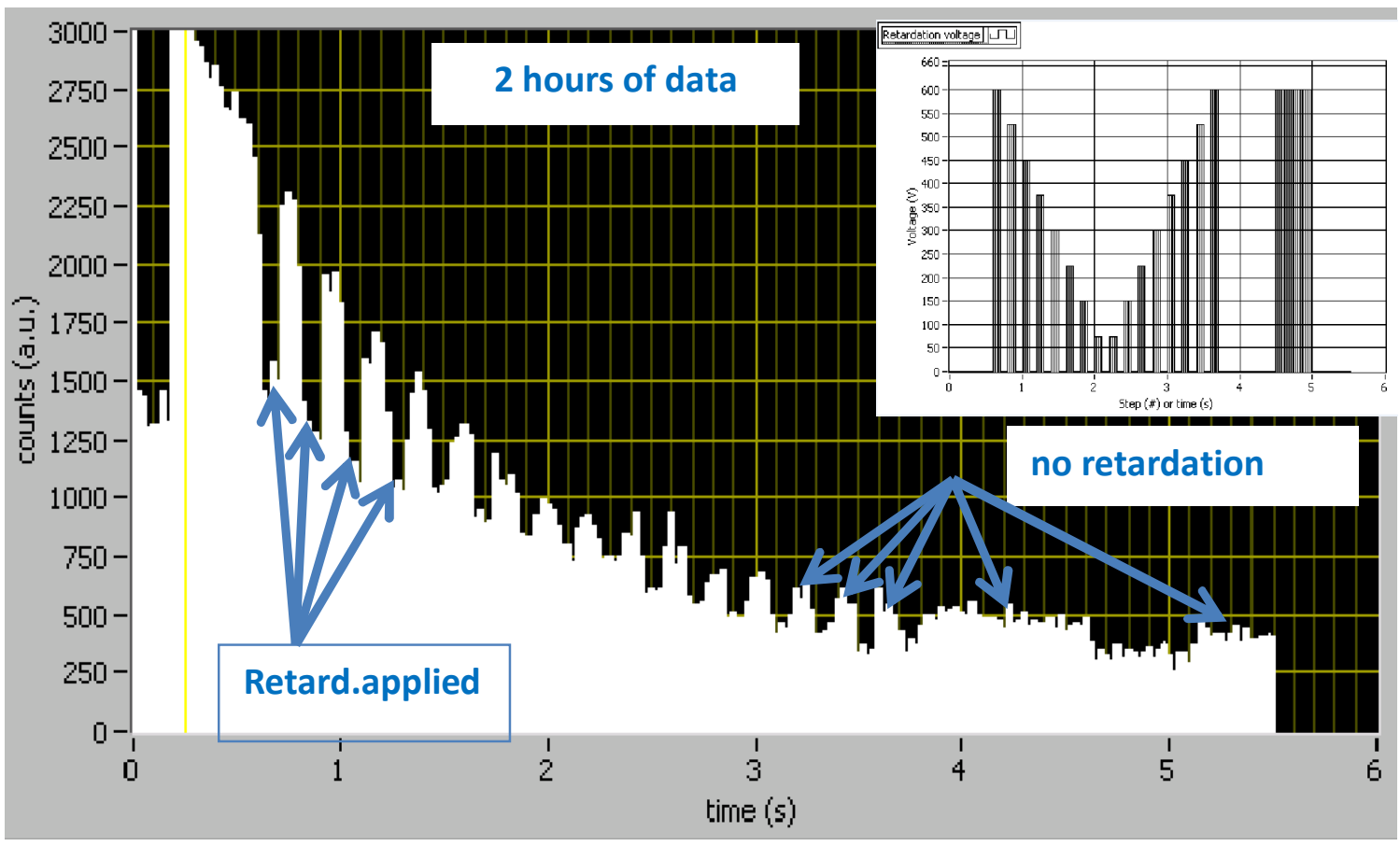

Fig. 4 Count rate in MCP detector within the measurement cycle with retardation pattern (see inset)

From the comparison of the unretarded spectrum (all ions getting to the MCP detector) and the retarded spectrum (only ions with energy higher than the retardation barrier) the integral recoil spectrum (Fig. 5) can be derived which allows determining the correlation coefficient $\boldsymbol{a}$ [9].

Fig.5 Experimental integral recoil spectrum of ${ }^{35} \mathrm{Ar}$

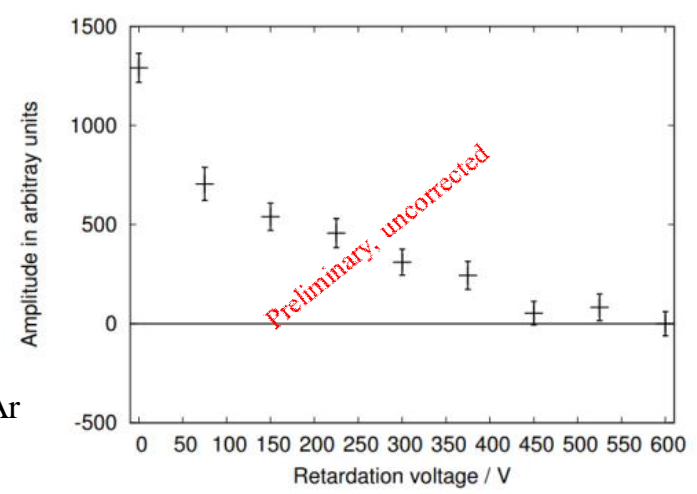




\subsection{Experiment in 2012}

A new online experiment was performed in 2012 using the experience gained from the experiment in 2011. We obtained more intensive and cleaner beam of ${ }^{35} \mathrm{Ar}$ from ISOLDE while the WITCH setup utilized better diagnostics, improved measurement system and methodics, better transmission and new DAQ enabling us to include more info into the written data and make the deadtime negligible. Only few levels of retardation potential were used in each measurement, while from the middle of the measuring cycle the ions were blocked and background was measured (Fig.6). In this way we achieved very important realtime monitoring of the background. The measured statistics was $\sim 10 \mathrm{x}$ higher than in the 2011 experiment.

Raw analysis of the experimental data was performed and raw recoil spectrum extracted (Fig.7) but full analysis is still continuing, systematic effects are studied/simulated and also some offline tests are still necessary to correct for some of them.

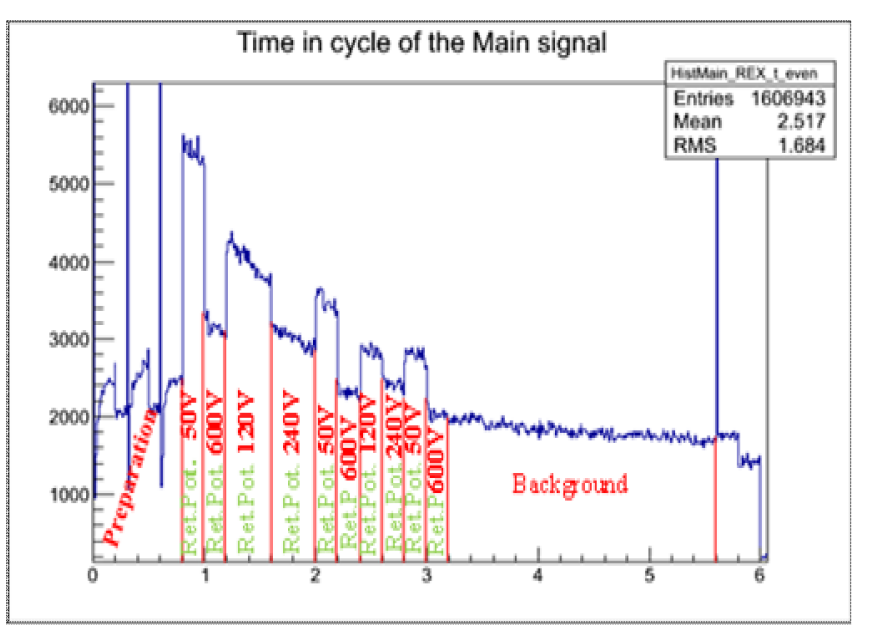

Fig.6 Measurement of the retardation spectrum of recoiling ions

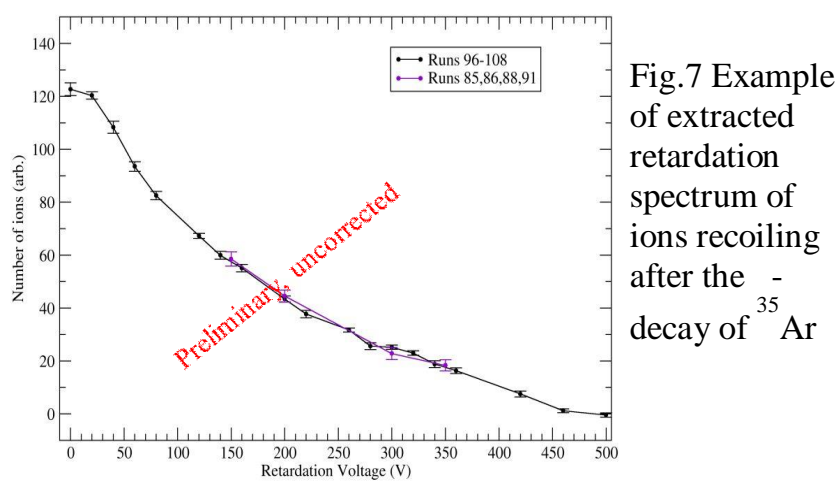

\section{Study of systematic effects}

\subsection{Ion clouds in Penning traps ï SIMBUCA simulations}

Ion bunches from REXTRAP (energy 30keV) are slowed down by the PulsedDriftTube [7] to $\sim 10^{1} \mathrm{eV}$, stopped in the ñcooler trapò and in He buffer gas cooled down to $\sim<10^{-1} \mathrm{eV}$. The high number of cooled ions (necessary to reach high statistics) have to be also well spatially compressed to fit to the narrow diaphragm between the traps $(\varnothing=2 \mathrm{~mm} \ddot{i}$ necessary to avoid leaking of buffer gas from cooler to decay trap) through which they are transferred into the decay trap. However, for higher ion densities the (Coulomb) selfinteractions of ions cannot be neglected. Therefore the simulation package Simbuca [10] was developed for simulations of the ion cloud behavior in ion traps (even for large numbers/densities of ions). This specialized

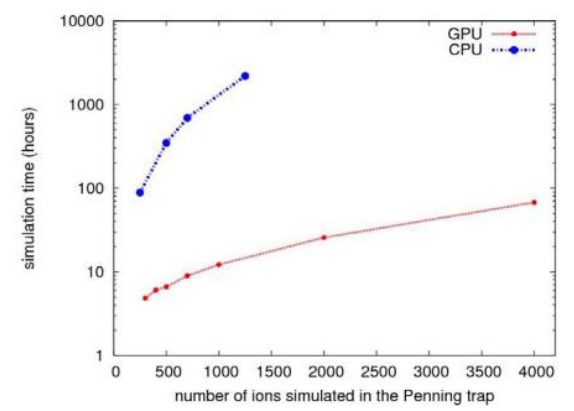

Fig.8 Comparison of the speed of calculations on CPU and GPU [10] 
program package calculates these ion self-interactions on the computer graphics card (GPU) contrary to standard programs running on the computer central processing unit (CPU). The usage of GPU paralellisation is absolutely essential ï long-range Coulomb interactions mean that each ion interacts with each other and standard calculations (using CPU) start to get unrealistic for $\sim 10^{3}$ ions (see Fig. 8) while we need to simulate $\sim>10^{5}$ ions in the trap.

The program simulates the space charge effects like the change of cyclotron and magnetron resonant frequency, change of the cloud shape and energy in traps due to self-interaction of ions, etc.. The ion selfinteractions (with high densities) seriously change the size and energy of the ion cloud then without these simulations our understanding of cloud properties is completely wrong. To illustrate this, Fig.9 shows how the cloud of ${ }^{35} \mathrm{Ar}$ ions expands in the cooler trap due to the Coulomb repulsion 1 i for $5^{*} 10^{6}$ ions and the initial cloud size $0.2 \mathrm{~mm}$ the self-repulsion blows up the ion cloud size after $200 \mathrm{~ms}$ by more than $300 \%$. A more detailed account will be given in [11].

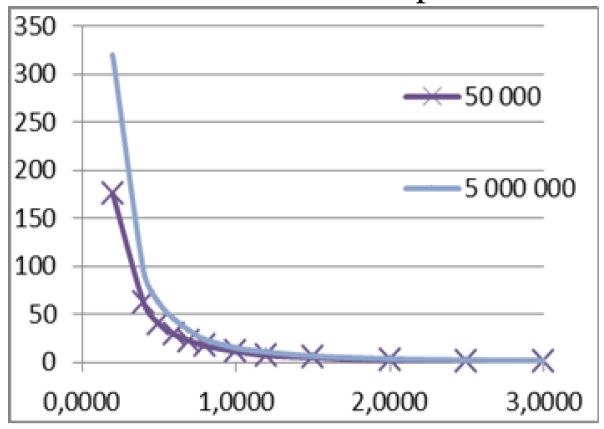

Fig.9 Relative increase of the cloud size [\%] depending on the initial cloud radius [mm] and number of ions $(50000,5000000)$

\subsection{Trajectories of recoiling ions ï SimWitch simulations}

The simulation package SimWitch [12] was developed to perform Monte Carlo simulations of the recoiling ion trajectories from the decay trap through the spectrometer to the MCP detector. Ion transport is simulated for various retardation voltages $(0 \mathrm{~V} \ddot{i} 450 \mathrm{~V})$ and various ${ }^{35} \mathrm{Cl}$ (recoiling ion after the ${ }^{35} \mathrm{Ar} \beta$-decay) charge states $\left(1^{+}, 2^{+}, 3^{+}, 4^{+}, 5^{+}\right)$. Original $2 \mathrm{D}$ version of the program (using cylindrical symmetry) was currently successfully upgraded to $3 \mathrm{D}$ (to account for the effect of several components in the setup breaking the cylindrical symmetry). Simulations are performed firstly for the optimization of parameters before the experiment and then for the analysis and correction of measured experimental data.

\subsection{MCP detector}

For the registration of recoil nuclei a position sensitive MCP detector [8] is used with the detection efficiency $40(11) \%$ which should be energy (and position) independent. Tests of resolution and efficiency of the MCP detector with perforated absorption mask and $\alpha$-particles ${ }^{241} \mathrm{Am}$ were done (Fig.10) prior to the online runs showing a good performance of the MCP detector.

Recently, the energy dependence of the MCP detector efficiency was measured as this could be a major systematic effect. The study of the energy dependence of the MCP detector efficiency for $\mathrm{Na}^{+}$ ions in energy range 0 ï $6.5 \mathrm{keV}$ was done at LPC Caen. Results showed that indeed the efficiency of

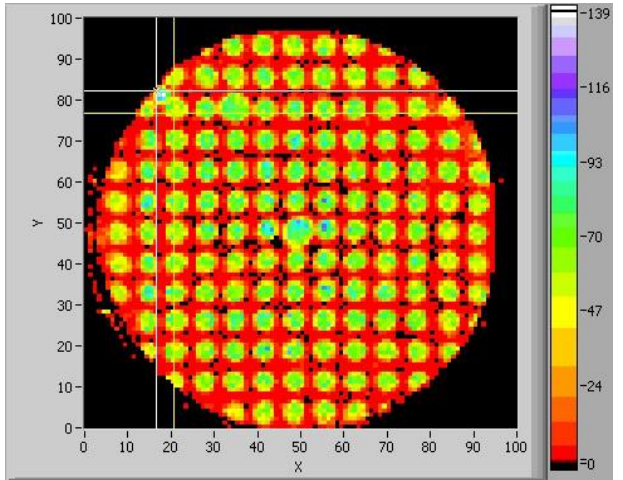

Fig.10 MCP detector with mask, ${ }^{241}$ Am $\alpha$ Q́ 


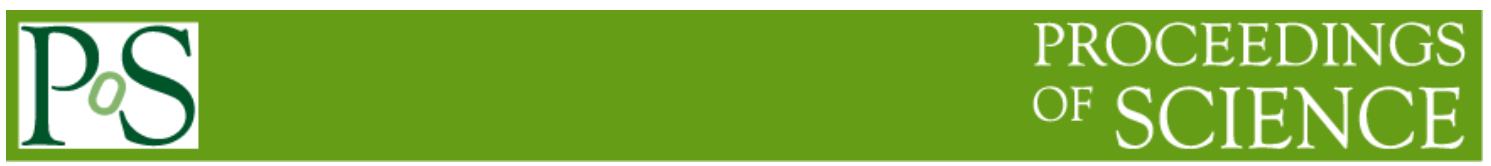

our MCP detector increases with ion energy in the region 3-6keV (Fig. 11) which is thus crucial systematic effect which needs to be corrected (higher charge states of $\beta$-decay products have higher energy due to reacceleration in front of the main $\mathrm{MCP} \Rightarrow$ energy dependent efficiency then deforms the charge state population). More detailed measurements of the efficiency of the MCP detector necessary for precise correction of experimental data are still planned.

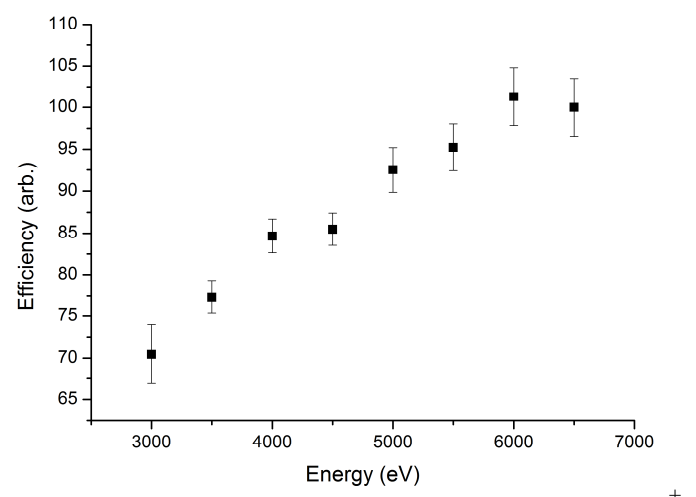

Fig.11 MCP detector efficiency for $\mathrm{Na}^{+}$ions

\section{Conclusion, outlook}

The experimental setup WITCH on the beam of ISOLDE works well - we are able to catch and cool ${ }^{35} \mathrm{Ar}$ ions in a combination of two Penning traps, keep them in traps with minimal energy $\left(\sim 10^{-1} \mathrm{eV}\right)$ for long enough time $(\sim>1 \mathrm{~s})$ with negligible losses and subsequently probe the energy of recoiling nuclei after the bï decay by means of a retardation spectrometer and MCP detector. The principle of this world unique setup is working - Penning traps are well suited to provide scattering-free radioactive sources for low energy spectrometry and the retardation spectrometer proved its suitability for studies of very low energies particles with high precision and efficiency.

Several online measurements of ${ }^{35} \mathrm{Ar}$ were performed at ISOLDE, retardation (recoil) spectra of ions produced in bï decay of ${ }^{35} \mathrm{Ar}$ were extracted and simulations are correctly describing the ions behaviour in traps and their tracking in the setup. First pilot experiment from 2011 with low statistics was fully analyzed and the value of angular $\beta-v$ correlation coefficient $\boldsymbol{a}$ was determined (albeit with a still rather large error bar) by the WITCH experiment [9]. Next online experiment from autumn 2012 with higher statistics, improved beam quality and measurement conditions is being analyzed, raw retardation spectrum is being extracted and systematic effects are studied.

We plan to finalise the analysis of the experimental data and extraction of recoil spectra, continue SimWITCH and Simbuca simulations for further understanding of the system and calculations of necessary systematic corrections to handle systematic uncertainties. More detailed experimental tests of the MCP detector efficiency will be done for precise correction of experimental data. Finally we plan to extract an experimental value of the $\beta-\nu$ correlation coefficient $\boldsymbol{a}$ with the best possible precision and compare it with its Standard Model value.

\section{References}

[1] R.D. McKeown and P. Vogel, Neutrino masses and oscillations: triumphs and challenges, Phys. Rep. 394 (2004) 315 
[2] C.S.Wu et al., Experimental Test of Parity Conservation in Beta Decay, Phys.Rev. 105(1957) 1413

[3] J.S.Allen et al., Determination of the Beta-Decay Interaction from Electron-Neutrino Angular Correlation Measurements, Phys.Rev. 116 (1959) 134

[4] N. Severijns, M. Beck, O. Naviliat-Cuncic, Tests of the standard electroweak model in nuclear beta decay, Rev. Mod. Phys. 78 (2006) 991

[5] N.Severijns et al., Search for New Physics in Beta-Neutrino Correlations using Trapped Ions and a Retardation Spectrometer, CERN-INTC-2005-014 ; CERN-INTC-2008-004

[6] M.Beck et al., WITCH: a recoil spectrometer for weak interaction and nuclear physics studies, NIM A503 (2003) 567

M.Beck et al., First detection and energy measurement of recoil ions following beta decay in a Penning trap with the WITCH experiment, Eur.Phys.J. A47 (2011) 45

[7] S.Coeck et al., A pulsed drift cavity to capture $30 \mathrm{keV}$ ion bunches at ground potential, NIM A572 (2007) 585

[8] S.Coeck et al., Microchannel plate response to high-intensity ion bunches, NIM A557 (2006) 516

E.Lienard et al., Performance of a micro-channel plates position sensitive detector, NIM A551 (2005) 375

[9] S.Van Gorp et al, First recoil-ion energy spectrum measurement of ions stored in a Penning trap, submitted to PRL

[10] S.VanGorp at al., Simbuca, using a graphics card to simulate Coulomb interactions in a penning trap, NIM A638(2011)192

[11] T.Porobic et al., Simulations for the behavior of many ions in a Penning trap, to be published

[12] P.Friedag, Setup and calibration of a position sensitive microchannel plate detector and analysis of a test run optimizing the WITCH experiment, PhD. Thesis, Univ. Of Muenster (2013) 